\title{
Assessing Depression in the Wild: Insights From Two Large-Scale Fully Mobile Randomized Clinical Trials
}

\author{
Abhishek Pratap $^{1 *}$, MS Eng; Joaquin A Anguera ${ }^{2 *}, \mathrm{PhD}$; Brenna N Renn ${ }^{1}, \mathrm{PhD}$; Joshua Volponi ${ }^{2}, \mathrm{PhD}$; David C \\ Atkins ${ }^{1}, \mathrm{PhD}$; Sean D Mooney ${ }^{1}, \mathrm{PhD}$; Pat A Areán ${ }^{1}, \mathrm{PhD}$
}

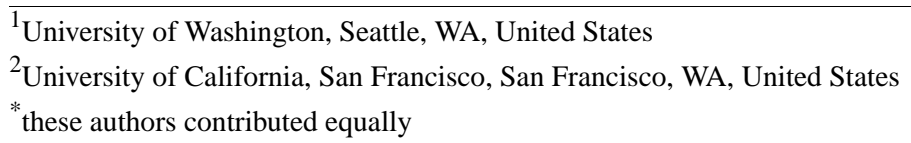

\section{Corresponding Author:}

Abhishek Pratap, MS Eng

University of Washington

Seattle, WA,

United States

Phone:

Email: apratap@uw.edu

\begin{abstract}
Background: The dispassionate ability of smartphones to stream real-time, personalized data from an array of onboard sensors can inform assessment and treatment of a range of medical conditions, including mental health issues. This technology can potentially address some of the known access barriers, including cost, time, stigma, shortage of mental health professionals, language barriers and other factors that prevent people from seeking help in a timely fashion. While this technology holds much promise in healthcare, its utility in describing patient phenotype from passive sensor data is unclear and an important area for research.
\end{abstract}

Objective: We will summarize the key learnings from two large-scale ( $>2,000$ enrolled people) fully mobile clinical trials targeting depressed individuals. Both studies were designed to assess the feasibility of running a remote, randomized controlled trial via custom applications. While BRIGHTEN v1 was open to the general US population, BRIGHTEN v2 was designed to enroll both English-speaking and an underserved Latino/Hispanic population. Here we will highlight the noticeable differences in user recruitment, engagement, and daily mood prediction observed across these studies, and explore the possible differences for each.

Methods: Both studies recruited participants through ads on Craigslist, targeted social media campaigns and other online classified-like resources. In total, 7,433 people responded and were screened for eligibility. Adults (18 years and older) with mild to severe depression as determined by a Patient Health Questionnaire [PHQ-9] score $\geq 5$ were eligible to join the study. There were 3,310 eligible participants, of which 2,176 were enrolled. Sensor-based data were collected passively, from typical smartphone usage (aggregated call logs and messaging history for people with Android-based phones, and GPS data for both iOS and Android devices). The main outcomes of the study were depressive symptomatology, quantified by the PHQ-9. Daily mood fluctuations were measured using a two-item depression questionnaire (PHQ-2).

Results: The overall compliance (the number of unique days an individual participates-i.e. completes active tasks) was drastically lower for V2 study with $<40 \%$ users completing any active task during the week 1 compared to $~ 80 \%$ in the V1 study. Considerable heterogeneity was seen amongst individuals when comparing the association of self-reported mood (PHQ-2/9 score) to various passive features, limiting the generation of a generalized cohort-level model for predicting mood (PHQ-2/9 score) based on passive features. Personalized models predicting mood at an individual level revealed a signal for some individuals (median R2 0.25). However, predictive power in the V1 study using individual digital behavior for four weeks was modest at best (median R2 0.15).

Conclusions: These studies demonstrate the feasibility of conducting large-scale mobile-based randomized trials and a marginal signal in the passive data to predict daily mood. However, further research is warranted to assess clinical relevance. Compliance was the primary factor affecting data analysis, and it limited our ability to draw inferences or employ predictive modeling at the cohort level. Studies are required to understand barriers and facilitators that are required to engage people, especially those with mental health issues. 
(iproc 2017;3(1):e46) doi: 10.2196/iproc.8504

\section{KEYWORDS}

Depression; Hispanic/Latino; minority group; Mobile health (mHealth); mobile health intervention; sensors; smartphone

\section{Multimedia Appendix 1}

Full poster.

[PDF File (Adobe PDF File), 3MB-Multimedia Appendix 1]

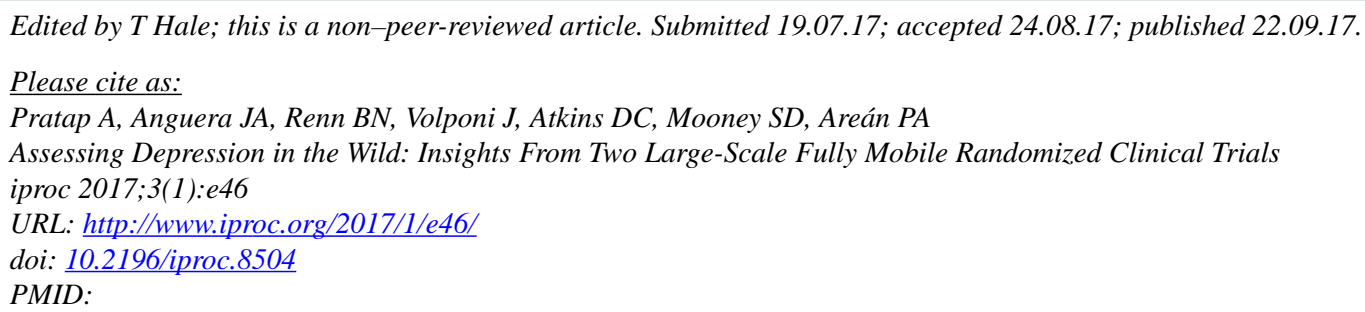

(C)Abhishek Pratap, Joaquin A. Anguera, Brenna N. Renn, Joshua Volponi, David C. Atkins, Sean D. Mooney, Pat A. Areán. Originally published in Iproceedings (http://www.iproc.org), 22.09.2017. This is an open-access article distributed under the terms of the Creative Commons Attribution License (https://creativecommons.org/licenses/by/4.0/), which permits unrestricted use, distribution, and reproduction in any medium, provided the original work, first published in Iproceedings, is properly cited. The complete bibliographic information, a link to the original publication on http://www.iproc.org/, as well as this copyright and license information must be included. 